\title{
Asymptotic stability of 3D functional Brinkman-Forchheimer equation
}

\author{
Xinguang Yang ${ }^{1}$, Yong Yang ${ }^{2}$, Wenjing Liu², and Junzhao Zhang ${ }^{3}$ \\ ${ }^{1}$ Henan Normal University \\ ${ }^{2}$ Beijing University of Technology \\ ${ }^{3}$ Changchun University of Technology
}

November 17, 2021

\begin{abstract}
This paper is concerned with the asymptotic stability of global weak and strong solutions for a 3D incompressible functional Brinkman-Forchheimer equation with delay. Under some appropriate assumptions on the external forces especially the averaged state, the well-posedness of 3D functional Brinkman-Forchheimer flow model and its steady state equation have been obtained rstly, then the asymptotic stability of global solutions also derived via the convergence of trajectories for the corresponding systems.
\end{abstract}

\section{Hosted file}

211027Asymptotic-BF.pdf available at https://authorea.com/users/315232/articles/545857asymptotic-stability-of-3d-functional-brinkman-forchheimer-equation 